\title{
Association Between Lipid Profiles and Left Ventricular Hypertrophy: New Evidence from a Retrospective Study
}

\author{
Xuewei Huang ${ }^{1,5 \dagger}$, Keqiong Deng ${ }^{2,3,4 \dagger}$, Juanjuan Qin ${ }^{1,5}$, Fang Lei ${ }^{5,6}$, Xingyuan Zhang ${ }^{5,6}$, \\ Wenxin Wang ${ }^{1,5}$, Lijin Lin ${ }^{1,5}$, Yuming Zheng ${ }^{7}$, Dongai Yao ${ }^{8}$, Huiming Lu' ${ }^{9}$, Feng Liu $^{10}$, \\ Lidong Chen ${ }^{11}$, Guilan Zhang ${ }^{12}$, Yueping Liu ${ }^{13,14}$, Qiongyu Yang ${ }^{15}$, Jingjing Cai ${ }^{16}$, \\ Zhigang She ${ }^{1,5 *}$, Hongliang $\mathrm{Li}^{1,3,5 *}$ \\ ${ }^{1}$ Department of Cardiology, Renmin Hospital of Wuhan University, Wuhan 430060, China \\ ${ }^{2}$ Department of Cardiology, Center Hospital of Huanggang, Huanggang, Hubei 438000, China \\ ${ }^{3}$ Huanggang Institute of Translation Medicine, Huanggang, Hubei 438000, China \\ ${ }^{4}$ Department of Cardiology, ${ }^{8}$ Physical Examination Center, Zhongnan Hospital of Wuhan University, Wuhan \\ 430071, China \\ ${ }^{5}$ Institute of Model Animal, ${ }^{6}$ School of Basic Medical Science, Wuhan University, Wuhan 430071, China \\ ${ }^{7}$ Physical Examination Center, The Central Hospital of Wuhan, Wuhan 430014, China \\ ${ }^{9}$ General Medical Department, CR \& WISCO General Hospital, Wuhan 430000, China \\ ${ }^{10}$ Information Center, Hubei Provincial Hospital of Integrated Chinese and Western Medicine, Wuhan \\ 430015, China \\ ${ }^{11}$ Department of Medical Examination Center, Taihe Hospital, Hubei University of Medicine, Shiyan, Hubei \\ 442000, China \\ ${ }^{12}$ Department of Health Management, Xiaogan Hospital Affiliated to Wuhan University of Science and \\ Technology, Xiaogan, Hubei 432000, China \\ ${ }^{13}$ Basic Medical Laboratory, General Hospital of Central Theater Command, Wuhan 430070, China \\ ${ }^{14}$ Hubei Key Laboratory of Central Nervous System Tumor and Intervention, Wuhan 430070, China \\ ${ }^{15}$ Chinese Medicine Center, Shiyan Renmin Hospital, Shiyan, Hubei 442000, China \\ ${ }^{16}$ Department of Cardiology, The Third Xiangya Hospital, Central South University, Changsha 410000, China
}

\begin{abstract}
Objective To explore the association between lipid profiles and left ventricular hypertrophy in a Chinese general population.

Methods We conducted a retrospective observational study to investigate the relationship between lipid markers [including triglycerides, total cholesterol, low-density lipoprotein cholesterol, high-density lipoprotein (HDL) cholesterol, non-HDL-cholesterol, apolipoprotein A-I, apolipoprotein B, lipoprotein[a], and composite lipid profiles] and left ventricular hypertrophy. A total of 309,400 participants of two populations (one from Beijing and another from nationwide) who underwent physical examinations at different health management centers between 2009 and 2018 in China were included in the cross-sectional study. 7,475 participants who had multiple physical examinations and initially did not have left ventricular hypertrophy constituted a longitudinal cohort to
\end{abstract}

Received January 22, 2022; accepted March 1, 2022; published online March 2, 2022.

*Corresponding author Hongliang Li, E-mail: lihl@whu.edu.cn, Tel: 86-27-68759302; Zhigang She, E-mail: zgshe@whu.edu.cn, Tel: 8627-68759885.

${ }^{\dagger}$ These authors have contributed equally to this work. 
analyze the association between lipid markers and the new-onset of left ventricular hypertrophy. Left ventricular hypertrophy was measured by echocardiography and defined as an end-diastolic thickness of the interventricular septum or left ventricle posterior wall $>11 \mathrm{~mm}$. The Logistic regression model was used in the cross-sectional study. Cox model and Cox model with restricted cubic splines were used in the longitudinal cohort.

Results In the cross-sectional study, for participants in the highest tertile of each lipid marker compared to the respective lowest, triglycerides [odds ratio (OR): 1.250, 95\% CI: 1.060 to 1.474 ], HDL-cholesterol (OR: 0.780, 95\% 0.662 to 0.918 ), and lipoprotein(a) (OR: $1.311,95 \% C I: 1.115$ to 1.541$)$ had an association with left ventricular hypertrophy. In the longitudinal cohort, for participants in the highest tertile of each lipid marker at the baseline compared to the respective lowest, triglycerides [hazard ratio (HR): 3.277, 95\% 1.720 to 6.244], HDL-cholesterol (HR: 0.516, 95\%CI: 0.283 to 0.940), non-HDL-cholesterol (HR: 2.309, 95\%CI: 1.296 to 4.112), apolipoprotein B (HR: 2.244, 95\%CI: 1.251 to 4.032) showed an association with new-onset left ventricular hypertrophy. In the Cox model with forward stepwise selection, triglycerides were the only lipid markers entered into the final model.

Conclusion Lipids levels, especially triglycerides, are associated with left ventricular hypertrophy. Controlling triglycerides level potentiate to be a strategy in harnessing cardiac remodeling but deserve to be further investigated.

Key words: left ventricular hypertrophy; lipid; triglycerides; apolipoprotein

\section{INTRODUCTION}

Left ventricular hypertrophy (LVH) is a structural change in the heart due to increased workload, which can be identified initially by a thickening of the ventricular wall ${ }^{[1]}$. It usually marks the occurrence of cardiac remodeling and is a marker of poor prognosis in cardiovascular diseases, such as heart failure ${ }^{[2,3]}$. Global societies are facing a dramatic increase in aging populations and its accompanied pandemic of metabolic diseases, particularly the increased disease burden associated with dyslipidemia ${ }^{[4]}$. As the fatty acids are the major energy substrates for cardiac muscle ${ }^{[5]}$, dyslipidemia may alter the metabolism in cardiomyocytes and result in LVH or other structural changes. However, there are few studies on the association between cardiac structural alterations and lipids, and controversial conclusions.

In previous small sample studies, cholesterol, triglycerides (TG), and high-density lipoprotein cholesterol (HDL-c) had all shown potential correlations with structural changes in the cardiac structures ${ }^{[6-10]}$. However, the findings have been inconclusive and most studies have been performed in hypertensive populations. Current studies have focused on a few limited lipid markers (cholesterol, HDL-c, triglycerides), and there are no intercomparisons between them. In addition, no large population studies explored the relationship between $\mathrm{LVH}$ and apolipoproteins (apolipoprotein A-I [ApoA-I], apolipoprotein B [ApoB]) and lipoprotein(a), and two cardiovascular risk factors have gained increasing attention in recent years ${ }^{[11]}$. Two small studies suggested that a composite, such as TG/
HDL-c may better reflect the association between lipids and LVH in children or general adults ${ }^{[12,13]}$. More importantly, there were no studies systematically discussed the relationship between blood lipids and LVH in the Chinese population. However, Asia, especially China, has been the most severely affected region for dyslipidemia in recent years and has a unique profile of risk factors for cardiac hypertrophy, which is characterized by hypertension, and other metabolic-related risk factors ${ }^{[14-17]}$. Therefore, it is essential to identify the association between lipids and cardiac remodeling in Chinese populations.

To clarify the association between lipid levels and $\mathrm{LVH}$ in the Chinese population, this study retrospectively collected data from 309,400 individuals who underwent both lipids profile analysis and transthoracic echocardiography in China, a total of 11 lipid markers included in our research. We also constructed a longitudinal cohort in those who underwent medical examinations at least twice with an interval of $>1$ year to further explore the association of lipids components with the new onset of LVH.

\section{SUBJECTS AND METHODS}

\section{Participants}

To analyze the association between lipid levels and echocardiography-reported LVH, we retrospectively collected health check-up records from individuals aged 18 years or older who underwent both lipid profile analysis [total cholesterol (TC), low-density lipoprotein cholesterol (LDL-c), HDL-C, TG, ApoA-I, ApoB, lipoprotein(a)] 
and transthoracic echocardiography at a health management center in Beijing, China, between October 2009 and November 2017. Individuals who undergo physical examinations were voluntarily at their own expense or the employer's expense. Initially, a total of 84,625 health check-up records were collected from 69,342 individuals. To reduce the confounding effects from other cardiac diseases, we excluded individuals with coronary artery disease, structural heart disease (valve stenosis, leakiness of heart valves, congenital heart disease, rheumatic heart disease, moderate and above or undetermined degree regurgitation, and other rare structural abnormalities), and those who had undergone cardiac surgery. Ultimately, 66,033 individuals were included in the Beijing cross-sectional population.

To increase the robustness of the results in the Beijing cross-sectional study, we constructed a national cross-sectional population. This population was from 24 health management centers in 10 provinces in China. The individuals aged 18 years or older were included in the analysis. All participants underwent transthoracic echocardiography and blood chemistry tests for LDL-C, HDL-C, TG, and TC. The exclusion criteria were consistent with those in the Beijing cross-sectional population. Finally, there were a total of 243,367 individuals recruited in the cross-sectional study nationwide (Fig. 1).

To further explore the relationship between lipid levels and the new onset of LVH during the follow-up, we recruited 7,475 participants from 8,843 who had multiple physical examinations with an interval of $>1$ year between physical examinations and did not have LVH at the first check-up constituted a longitudinal cohort (Fig. 1).

The data for this study was based on a nationwide research project on physical examination data initiated by the Renmin Hospital of Wuhan University, which was approved by the ethics committee of the Renmin Hospital of Wuhan University and subsequently by the ethics committees at each partner hospital. Informed consent was waived because no harm would be caused to the participants while no traceable personal information was included. This study followed the STrengthening the Reporting of OBservational studies in Epidemiology (STROBE) statement.

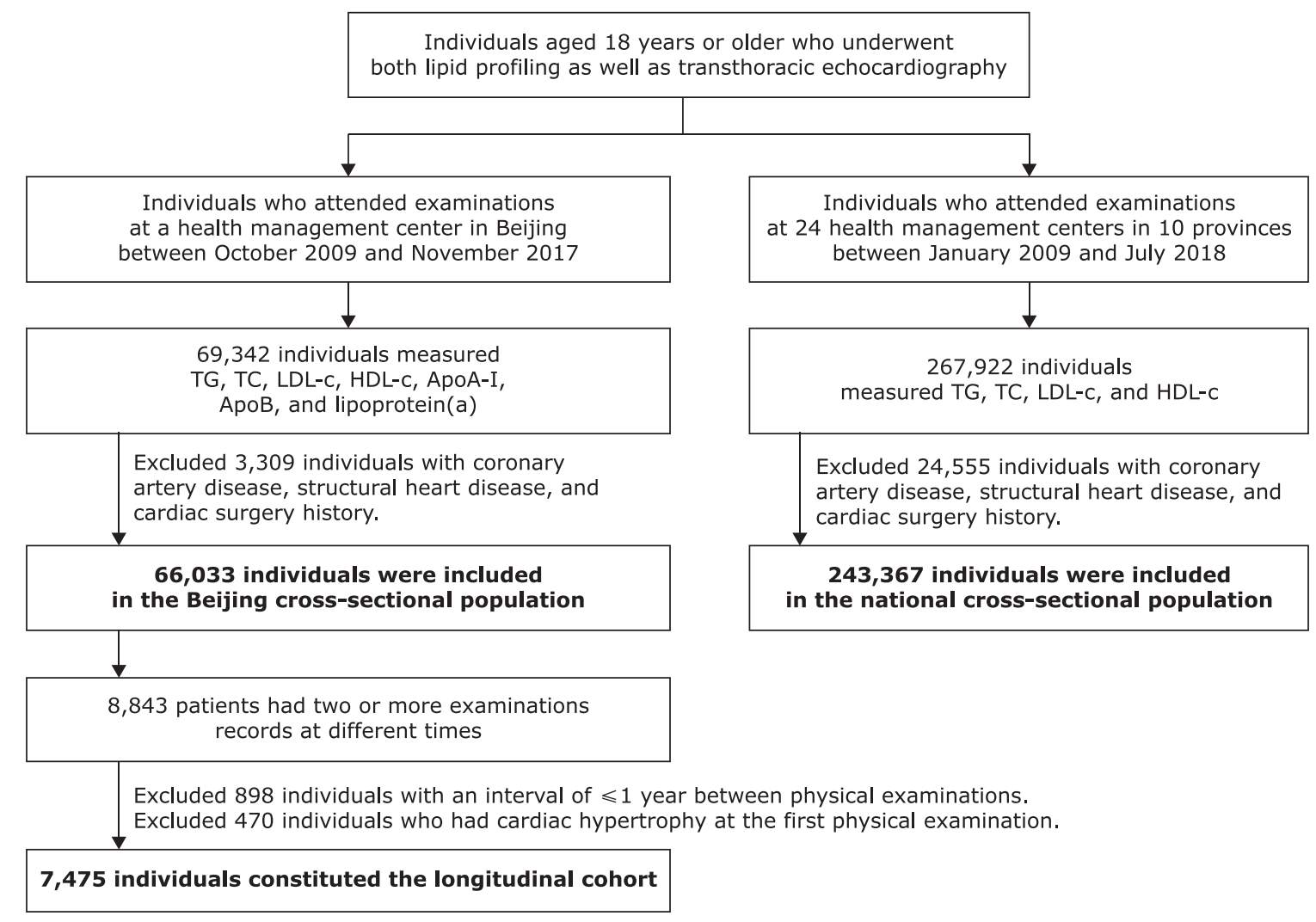

Figure 1. Flowchart of participant inclusion and exclusion.

TG: triglycerides; TC: total cholesterol; HDL-c: high-density lipoprotein cholesterol; LDL-c: low-density lipoprotein cholesterol; ApoB: apolipoprotein B; ApoA-I: apolipoprotein A-I. 


\section{Key measurements and definition}

Changes in the cardiac structure were measured by transthoracic M-mode echocardiography. Echocardiography was performed by experienced physicians, and we collected the final text results of ultrasound diagnostic. The diagnosis for LVH was based on the report of echocardiography. All health check-up centers followed the identical guidance for diagnosing LVH, namely that the end-diastolic thickness of the interventricular septum or/and left ventricle posterior wall reported above $11 \mathrm{~mm}^{[18,19]}$.

Lipid contents, including LDL-C, HDL-C, TG, TC, ApoB, ApoA-I, and lipoprotein(a) were measured directly according to Chinese guideline recommendations ${ }^{[20]}$. Detailly, TG and TC were measured by the enzymatic method, HDL-C and LDL-C by the homogeneous method, and ApoA-I, ApoB, and lipoprotein(a) by immunoturbidimetric method. Non-HDL-c was calculated by TC minus HDL-C. The composite parameters, including TG/HDL-C, TC/HDL-C, and ApoB/ApoA-I, were calculated accordingly based on the available indices.

\section{Anthropometric and laboratory data}

All participants had undergone comprehensive anthropometric measurements and clinical examinations by professional and experienced medical teams in each hospital.

Weight and height were measured to the nearest $0.1 \mathrm{~kg}$ and $0.1 \mathrm{~cm}$, respectively, in subjects wearing light clothing without shoes. Body mass index (BMI) was calculated by weight $(\mathrm{kg}) /$ height $^{2}\left(\mathrm{~cm}^{2}\right)$. Obesity was determined regarding Chinese standard weight (normal: BMI < 24, overweight: BMI $\geqslant 24$ and $<28$, obese: $B M I \geqslant 28)^{[21]}$.

Systolic blood pressure (SBP, $\mathrm{mm} \mathrm{Hg}$ ) was measured by mercury sphygmomanometers or electronic sphygmomanometers after individuals rested in a seated position for a minimum of $5 \mathrm{~min}$. Hypertension and diabetes were diagnosed based on personal history and Chinese hypertension or diabetes guideline ${ }^{[22,23]}$.

Blood routine tests and biochemical tests were conducted in fasting state measured according to standard protocols and guidelines at a certified laboratory, and all test results were obtained by the automated biochemical analyzer. In detail, we collected the results of blood regular test [including white blood cells, hemoglobin, red blood cells (RBC), and platelets], liver function test [including alanine transaminase (ALT), aspartate transaminase, gamma-glutamyl transpep- tidase, and albumin], renal function test [including serum creatinine, blood urea nitrogen (BUN), and eGFR (calculated by CKD-EPI formula)], inflammatory marker [C-reactive protein (CRP)], blood glucose level and pancreatic function test (including fasting glucose level, insulin C-peptide, insulin level, and hemoglobin A1c). Insulin resistance index (HOMA-IR) was calculated by fasting insulin $(\mathrm{mIU} / \mathrm{L}) \times$ fasting blood glucose $(\mathrm{mmol} / \mathrm{L}) / 22.5^{[24]}$.

\section{Data missing and imputation}

The average missing rate was $2.36 \%$ and the highest missing rate was $9.60 \%$ for the Beijing cross-sectional population and the longitudinal cohort from Beijing. The average and the highest missing rates were $4.05 \%$ and $22.19 \%$ in the cross-sectional population from nationwide. We used nonparametric missing value imputation which is based on the missForest procedure in $\mathrm{R}$ to fill in the missing data ${ }^{[25,26]}$. The results of the subsequent analysis of the dataset before and after imputation were not significantly different.

\section{Statistical methods}

In this study, continuous variables were expressed as medians and quartiles. Categorical variables were expressed as percentages. Mann-Whitney $U$ test was used for comparison of continuous variables and chisquare test was used for categorical variables. We used Spearman rank correlation analysis to analyze the correlation between lipid profiles. Unless specified, two-sided $P$-values $<0.05$ were considered statistically different in this study.

We used the Logistic regression model in cross-sectional data to analyze the relationship between lipid levels as categorical variables and LVH. In the Beijing cross-sectional population, we performed the univariable Logistic regression model at first and variables with $P$-values $<0.1$ were considered as potential covariates to be included in the next multivariable regression model. Meanwhile, we also considered the clinical significance of the variables and carefully identified the final covariates to ensure the simplicity of the multivariable regression model. Variables, including age, sex, BMI, SBP, CRP, RBC, ALT, BUN, and In(HOMA-IR) were considered as covariates. Since insulin resistance has a significant impact on the metabolism of lipids and cardiomyocytes ${ }^{[27,28]}$, this interaction was also adjusted by including lipid levels $x$ insulin resistance as cofounders into the models. In the 
national cross-sectional population, we validated the relationship between lipid profiles and LVH by Logistic regression model with covariates including age, sex, BMI, SBP, fasting glucose, ALT, hemoglobin, and BUN.

Cox regression model was used to identify the relationship between lipid levels and the new onset of LVH in the longitudinal cohort. We first performed a univariable Cox regression analysis to select variables for the following multivariable Cox regression. The variables, including age, sex, BMI, SBP, RBC, ALT, BUN, and In(HOMA-IR), were adjusted as covariates. In the multivariable Cox model, the interaction of insulin resistance with lipids was also controlled. In addition, we put all lipids and the aforementioned covariates simultaneously into the Cox model with forward stepwise selection based on likelihood ratio to find the most important lipid profiles. We tested all Cox models for compliance with the proportional hazard assumption by Schoenfeld residuals method and found no violations (all $P$-values $>0.05$ ).

We also explored the relationship between lipid levels and the occurrence of LVH using the Cox regression model with restricted cubic splines (RCS) in the longitudinal cohort. The 10th, 50th, and 90th percentiles were set as nodes, and the median was the reference value. The top $1 \%$ of the result of each lipid marker was removed to exclude the effect of extreme values.

Statistical analysis for this study was performed using SPSS Statistics (version 26.0, IBM, Armonk, NY, USA) as well as R (version 3.6.3, R Foundation for Statistical Computing, Vienna, Austria).

\section{Sensitivity test}

To test the robustness of the results of the longitudinal cohort study, we performed three sensitivity tests. In the first sensitivity test, lipids were included in the models as continuous variables after being standardized. Second, we selected participants who had multiple physical examinations with an interval of $>2$ years between physical examinations and did not have LVH at the first check-up constituted a longitudinal cohort. Due to the limitation of the number of participants who happened $\mathrm{LVH}$, lipid markers were included in the models as continuous variables after being standardized instead of categorical variables to ensure the accuracy of statistics. Third, we excluded participants with hypertension at the baseline to reduce the influence of hypertension on LVH.

\section{RESULTS}

\section{Characteristics of the Beijing cross-sectional population}

The demographic characteristics of the Beijing cross-sectional population was presented in Table 1. A total of 66,033 participants were included in the Beijing cross-sectional study, of whom 4,351 (6.60\%) had a physical examination record reporting increased LV wall thickness. Among the included participants, $64.99 \%$ were men and the median age was 49 (IQR: 43 to 54 ). There were $19.74 \%$ participants with obesity and $23.51 \%$ with hypertension. In the Beijing cross-sectional population, individuals with echocardiography reported LVH had a significantly higher age (median: 52 versus 48, $P<0.001$ ) and proportion of males $(87.47 \%$ versus $63.40 \%, P<0.001)$. Also, the TG levels (median: $1.79 \mathrm{mmol} / \mathrm{L}$ versus $1.44 \mathrm{mmol} / \mathrm{L}$, $P<0.001)$ and the prevalence of obesity $(44.19 \%$ versus $18.02 \%, P<0.001$ ), hypertension ( $52.25 \%$ versus $21.48 \%, P<0.001)$, and diabetes mellitus (31.69\% versus $14.46 \%, P<0.001$ ) were significantly higher than those in participants without echocardiography reported LVH (Table $\mathbf{1}$ ).

\section{Association between lipids and LVH in the Beijing cross-sectional population}

We performed Logistic regression in the Beijing cross-sectional population to explore the association between lipid markers and LVH. The odds ratio $(O R)$ after adjusted age, sex, BMI, SBP, CRP, RBC, ALT, BUN, In(HOMA-IR), and the interaction between In(HOMA-IR) and lipids were shown in Fig. 2.

In this population, the highest tertiles of TG and lipoprotein(a) were significantly associated with a higher risk of presenting LVH compared with the respective lowest tertiles (TG, OR: 1.250,95\%CI: 1.060 to 1.474 ; lipoprotein[a], OR:1.311, 95\%CI: 1.115 to 1.541 ). And the second tertiles also had higher risk versus the lowest tertiles respectively (TG, OR: $1.249,95 \% C I$ : 1.068 to 1.462 ; lipoprotein[a], OR: $1.303,95 \% C I$ : 1.107 to 1.534$)$. In contrast, TC, LDL-C, non-HDL-C, and $A p o B$ levels did not have a significant association with the presence of LVH.

Participants in the highest tertile HDL-c had a 22\% (OR: $0.780,95 \% C I: 0.662$ to 0.918 ) reduction in $\mathrm{LVH}$ risk compared with the lowest tertile. Although ApoA-I is the major protein component of $\mathrm{HDL}$, which is involved in cholesterol traffic and mobilization ${ }^{[11]}$, 
and the levels of the two entities have a high correlation (Supplementary Table S1), the ApoA-I level was not associated with the presence of LVH in the cross-sectional study.

For composite lipid profiles, individuals in higher tertile of TC/HDL-C (OR: $1.283,95 \%$ CI: 1.089 to 1.510 ) and TG/HDL-C (OR: $1.288,95 \% C I: 1.092$ to 1.520 ) were significantly associated with the presence of LVH. But ApoB/ApoA-I levels were not shown a significant association with LVH.

Table 1. Characteristics of the Beijing cross-sectional population [median (IQR)]

\begin{tabular}{|c|c|c|c|c|c|}
\hline Items & Overall & Normal LV wall thickness & LV wall thickening & $z$ or $\chi^{2}$ & $P$ \\
\hline$n$ & 66,033 & $61,682(93.40 \%)$ & $4,351(6.60 \%)$ & & \\
\hline Age (yrs) & $49(43,54)$ & $48(43,54)$ & $52(47,59)$ & 27.46 & $<0.001$ \\
\hline Women $[n(\%)]$ & $23,118(35.01)$ & $22,573(36.60)$ & $545(12.53)$ & $1,034.90$ & $<0.001$ \\
\hline BMI $\left(\mathrm{kg} / \mathrm{m}^{2}\right)$ & $25.18(22.97,27.38)$ & $25.00(22.83,27.18)$ & $27.52(25.61,29.64)$ & 46.51 & $<0.001$ \\
\hline Obesity $[n(\%)]$ & $11,512(19.74)$ & $9,811(18.02)$ & $1,701(44.19)$ & $1,554.67$ & $<0.001$ \\
\hline Systolic blood pressure $(\mathrm{mmHg})$ & $121(109,134)$ & $120(108,133)$ & $136(125,150)$ & 49.79 & $<0.001$ \\
\hline Hypertension [n(\%)] & $13,839(23.51)$ & $11,808(21.48)$ & $2,031(52.25)$ & $1,911.29$ & $<0.001$ \\
\hline Total cholesterol (mmol/L) & $4.75(4.17,5.39)$ & $4.75(4.17,5.39)$ & $4.79(4.16,5.46)$ & 2.12 & 0.034 \\
\hline LDL-cholesterol (mmol/L) & $3.06(2.53,3.61)$ & $3.06(2.53,3.61)$ & $3.07(2.50,3.63)$ & -0.81 & 0.417 \\
\hline HDL-cholesterol ( $\mathrm{mmol} / \mathrm{L})$ & $1.17(0.97,1.41)$ & $1.17(0.98,1.42)$ & $1.06(0.89,1.26)$ & -24.86 & $<0.001$ \\
\hline Non-HDL-cholesterol (mmol/L) & $3.54(2.95,4.17)$ & $3.53(2.94,4.16)$ & $3.70(3.10,4.33)$ & 11.08 & $<0.001$ \\
\hline Triglycerides (mmol/L) & $1.46(1.01,2.19)$ & $1.44(0.99,2.16)$ & $1.79(1.27,2.73)$ & 24.92 & $<0.001$ \\
\hline Total cholesterol/HDL-c ratio & $4.05(3.27,4.97)$ & $4.02(3.24,4.93)$ & $4.50(3.72,5.40)$ & 23.44 & $<0.001$ \\
\hline Triglycerides/HDL-c ratio & $1.26(0.76,2.13)$ & $1.23(0.74,2.08)$ & $1.71(1.06,2.82)$ & 27.97 & $<0.001$ \\
\hline Apolipoprotein B (g/L) & $0.91(0.77,1.07)$ & $0.91(0.76,1.06)$ & $0.94(0.80,1.10)$ & 9.64 & $<0.001$ \\
\hline Apolipoprotein A-I (g/L) & $1.31(1.16,1.48)$ & $1.32(1.16,1.49)$ & $1.27(1.13,1.43)$ & -11.01 & $<0.001$ \\
\hline ApoB/ApoA-I ratio & $0.70(0.56,0.85)$ & $0.69(0.56,0.84)$ & $0.74(0.61,0.88)$ & 14.30 & $<0.001$ \\
\hline Lipoprotein(a) (mg/L) & $119.00(45.60,261.00)$ & $119.00(45.30,262.00)$ & $119.00(50.00,254.00)$ & -0.51 & 0.610 \\
\hline Uric acid $(\mu \mathrm{mol} / \mathrm{L})$ & $337.00(274.00,399.00)$ & $333.00(271.00,396.10)$ & $376.00(321.00,436.00)$ & 30.42 & $<0.001$ \\
\hline Hemoglobin A1c (\%) & $5.60(5.30,5.90)$ & $5.50(5.30,5.90)$ & $5.80(5.50,6.40)$ & 29.43 & $<0.001$ \\
\hline Fasting blood-glucose (mmol/L) & $5.30(4.92,5.86)$ & $5.28(4.90,5.81)$ & $5.77(5.24,6.75)$ & 36.27 & $<0.001$ \\
\hline Diabetes mellitus $[n(\%)]$ & $10,284(15.60)$ & $8,906(14.46)$ & $1,378(31.69)$ & 915.73 & $<0.001$ \\
\hline C-reactive protein $(\mathrm{mg} / \mathrm{L})$ & $1.20(0.70,2.10)$ & $1.20(0.70,2.00)$ & $1.60(0.90,2.80)$ & 19.08 & $<0.001$ \\
\hline Hemoglobin $(\mathrm{g} / \mathrm{L})$ & $147.00(134.00,157.00)$ & $146.00(134.00,157.00)$ & $152.00(143.00,160.00)$ & 22.72 & $<0.001$ \\
\hline Red blood cell $\left(\times 10^{12} / L\right)$ & $4.77(4.43,5.09)$ & $4.76(4.42,5.09)$ & $4.92(4.61,5.19)$ & 19.80 & $<0.001$ \\
\hline White blood cell $\left(\times 10^{9} / L\right)$ & $5.87(4.98,6.92)$ & $5.85(4.96,6.90)$ & $6.15(5.25,7.20)$ & 12.15 & $<0.001$ \\
\hline Alanine transaminase (IU/L) & $19.95(14.10,29.50)$ & $19.70(13.90,29.10)$ & $23.95(17.10,34.80)$ & 21.77 & $<0.001$ \\
\hline Aspartate transaminase (IU/L) & $18.40(15.40,22.70)$ & $18.40(15.40,22.60)$ & $19.40(16.10,24.90)$ & 13.07 & $<0.001$ \\
\hline GGT (IU/L) & $28.00(17.00,49.00)$ & $27.00(16.30,48.00)$ & $39.00(25.00,69.00)$ & 31.22 & $<0.001$ \\
\hline Serum creatinine $(\mu \mathrm{mol} / \mathrm{L})$ & $67.00(57.00,77.00)$ & $67.00(57.00,77.00)$ & $71.00(63.00,80.00)$ & 20.65 & $<0.001$ \\
\hline eGFR $\left[\mathrm{ml} /\left(\min \cdot 1.73 \mathrm{~m}^{2}\right)\right]$ & $105.72(95.24,112.34)$ & $105.90(95.81,112.48)$ & $102.89(81.83,110.31)$ & -16.40 & $<0.001$ \\
\hline Albumin (g/L) & $45.00(42.60,47.30)$ & $45.00(42.60,47.30)$ & $45.20(42.70,47.20)$ & 1.30 & 0.194 \\
\hline Blood urea nitrogen $(\mathrm{mmol} / \mathrm{L})$ & $5.00(4.30,5.90)$ & $5.00(4.30,5.90)$ & $5.30(4.50,6.20)$ & 16.41 & $<0.001$ \\
\hline Platelet count $\left(\times 10^{9} / \mathrm{L}\right)$ & $220.00(187.00,256.00)$ & $220.00(187.00,257.00)$ & $209.00(177.00,244.00)$ & -13.53 & $<0.001$ \\
\hline C-peptide (ng/ml) & $2.42(1.88,3.09)$ & $2.38(1.85,3.04)$ & $3.00(2.39,3.73)$ & 38.11 & $<0.001$ \\
\hline Fasting insulin (mU/L) & $9.22(6.38,13.36)$ & $9.07(6.28,13.08)$ & $11.87(8.29,17.14)$ & 29.86 & $<0.001$ \\
\hline HOMA-IR & $2.28(1.48,3.54)$ & $2.27(1.47,3.50)$ & $2.50(1.63,3.98)$ & 10.17 & $<0.001$ \\
\hline
\end{tabular}

LV: left ventricular; BMI: body mass index; LDL: low-density lipoprotein; HDL: high-density lipoprotein; GGT: gammaglutamyl transpeptidase; eGFR: estimated glomerular filtration rate; HOMA-IR: insulin resistance index.

$P$ values were calculated by Mann-Whitney $U$ test for continuous variables and Chi-square test for categorical variables. 


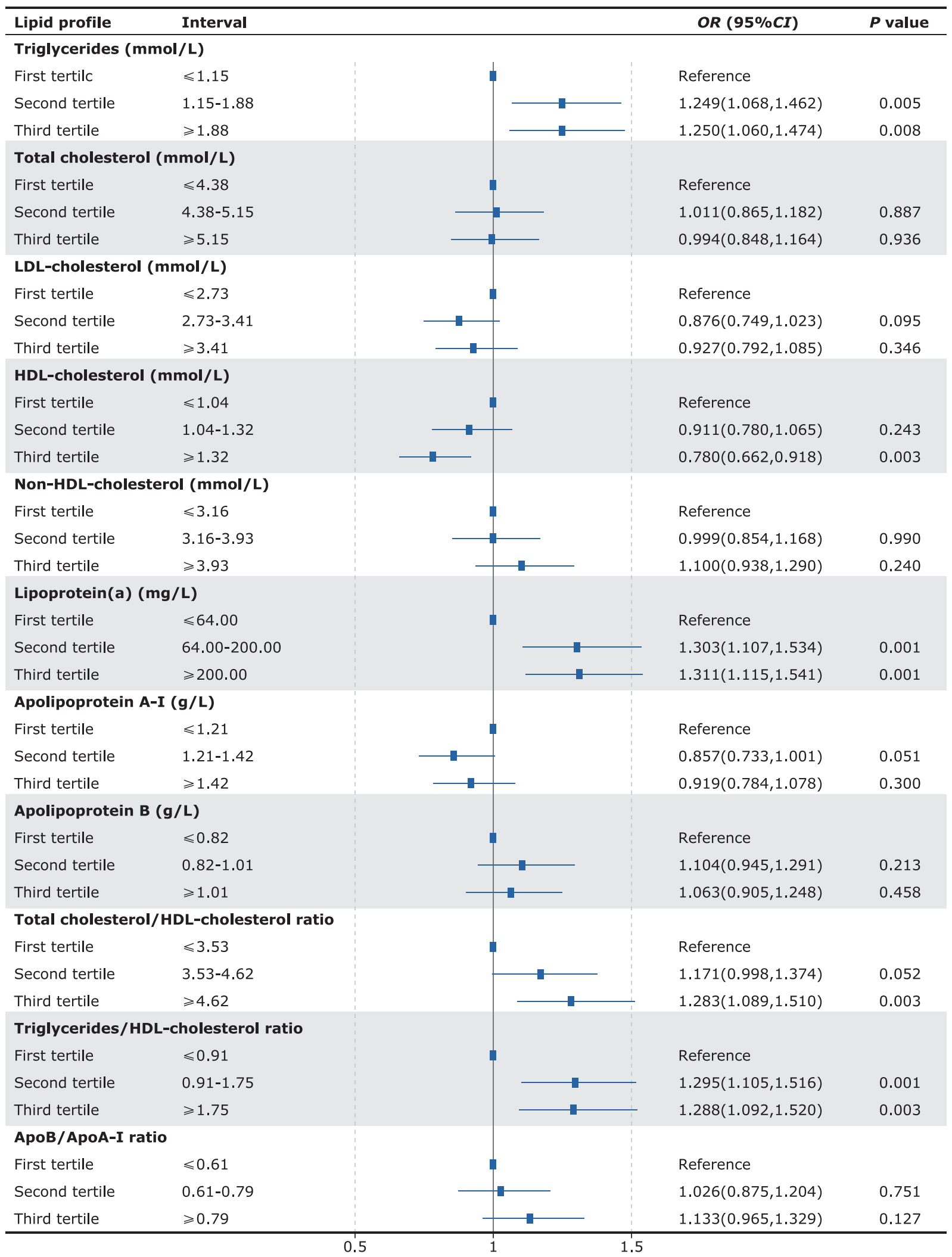

Figure 2. Relationship between lipid profiles and cardiac hypertrophy in cross-sectional analysis.

OR: odds ratio.

The adjustment factors of logistic model include age, sex, BMI, systolic blood pressure, C-reactive protein, red blood cell count, alanine transaminase, blood urea nitrogen, In(HOMA-IR), and the interaction between $\ln ($ HOMA-IR) and lipids. 
Association between lipids and LVH in the national cross-sectional population

To increase the robustness of the results of the Beijing cross-sectional population, we constructed a population which participants from nationwide. The effect of ApoA-I, ApoB, and lipoprotein(a) was not further addressed in this population due to limitations in data availability.

A total of 243,367 ( $62.16 \%$ of male) participants were included in this population. Overall, the characteristics of the national cross-sectional population had similar results with the Beijing cross-sectional population (Supplementary Table S2), but the proportion of echocardiography reported LVH was lower $(6,075$ participants, $2.50 \%$ ) in this population.

We performed Logistic regression in the national cross-sectional population with covariates including age, sex, BMI, SBP, fasting glucose, ALT, hemoglobin, and BUN (Supplementary Fig. S1). TG, HDL-C, TC/ HDL-C, and TG/HDL-C, which showed statistically significant associations with LVH in the Beijing population, also showed significant associations with LVH in this population. The result of LDL-C was consistent with the Beijing cross-sectional population as well. Statistically significant positive associations were observed when TC and non-HDL-c levels reached the highest tertile in the national cross-sectional population with more participants.

\section{Baseline characteristics of the longitudinal cohort} 7,475 participants with two or more health check-up records with an interval of > 1 year between examinations and normal ventricular wall thickness at the first examination constituted the longitudinal cohort in Beijing. The median follow-up time of the cohort was 913 (IQR: 622 to 1,387) days, and 307 (4.11\%) individuals newly reported LVH throughout the follow-up period. The baseline characteristics of the cohort population were similar to the Beijing cross-sectional population due to the cohort population was derived from it. The median age of the cohort was younger (median: 47 versus 49) because only participants initially without LVH were included (Supplementary Table S3).

\section{Association between lipids and new-onset LVH in the longitudinal cohort analysis}

We further addressed the association between lipid markers and new-onset LVH by a longitudinal cohort with 7,475 participants. We performed the analysis using the Cox model with covariates including age, sex, BMI, SBP, RBC, ALT, BUN, In(HOMA-IR), and the interaction between $\ln (\mathrm{HOMA}-\mathrm{IR})$ and lipid markers, the results are shown in Fig. 3.

In the longitudinal cohort, individuals with the second (HR: $3.838,95 \% C I: 2.070$ to 7.118 ) and highest tertile (HR: $3.277,95 \% C I: 1.720$ to 6.244 ) of TG level had over 3 -folds higher risk of new-onset LVH compared to those with TG levels in the lowest tertile. Furthermore, we visualized the relationship between TG and the risk of developed LVH using RCS in the longitudinal cohort, we found the risk had rapidly increased until TG reached $3 \mathrm{mmol} / \mathrm{L}$ (Fig. 4). Participants who were with non-HDL-c or ApoB at the highest tertile compared who were at the lowest tertile had an increased risk of new incident LVH during the follow-up. In contrast, the TC, LDL-C, and lipoprotein(a) levels did not demonstrate a positive correlation with new-onset LVH.

HDL-c in the highest tertile at baseline was associated with a decreased risk of LVH incidence in the longitudinal cohort. The RCS results showed a linear and negative association between increased HDL-C and the risk of new-onset LVH during the follow-up (Fig. 4). However, the level of ApoA-I level was not associated with the incidence of $\mathrm{LVH}$ in the longitudinal cohort. The RCS results suggest that ApoA-I has a similar but flatter relationship curve versus HDL-c (Fig. 4).

In addition, all three composite lipid markers (TC/ HDL-C, TG/HDL-C, and ApoB/ApoA-I) showed a positive correlation with the new-onset LVH risk in the longitudinal cohort.

Among lipid markers and lipoproteins that have a significant association with the new-onset LVH (TG, HDL-C, ApoB, non-HDL-C, TC/HDL-C, TG/HDL-C, and ApoB/ApoA-I) in the longitudinal cohort, we further determined which lipid marker has the strongest association with the incidence of new-onset LVH using the Cox model with forward stepwise selection based on likelihood ratio. TG was the only lipid that entered the model, which indicated TG was the most relevant indicator for subsequent development of LVH (Table 2).

\section{Sensitivity analyses for the longitudinal cohort} We further performed three separate sensitivity analyses to validate the robustness of the cohort results by changing the type of variable for lipids, increasing 


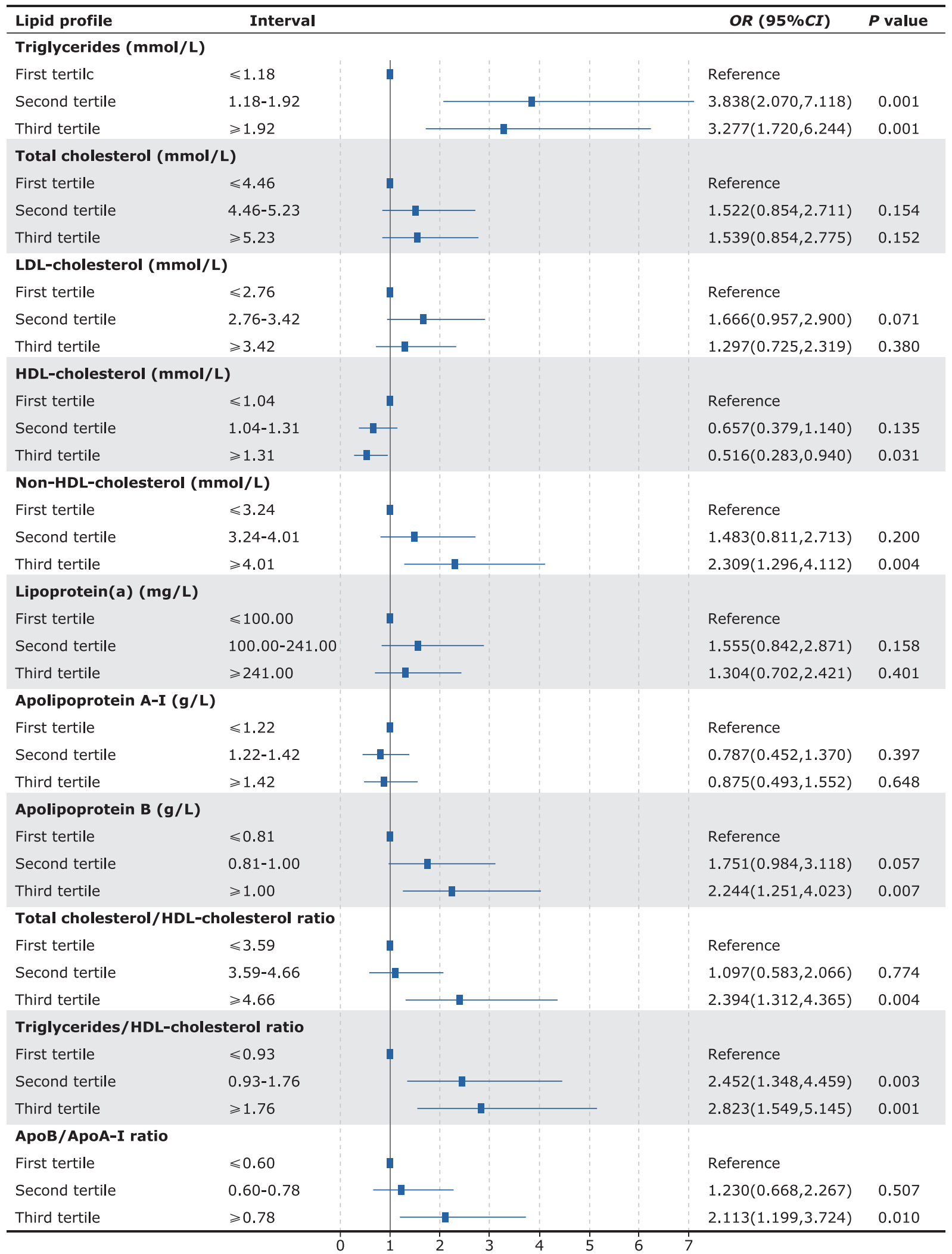

Figure 3. Relationship between lipid profiles and cardiac hypertrophy in cohort analysis.

HR: hazard ratio.

The adjustment factors of the Cox model include age, sex, BMI, systolic blood pressure, red blood cell count, alanine transaminase, blood urea nitrogen, $\ln ($ HOMA-IR), and the interaction between $\ln ($ HOMA-IR) and lipids. 

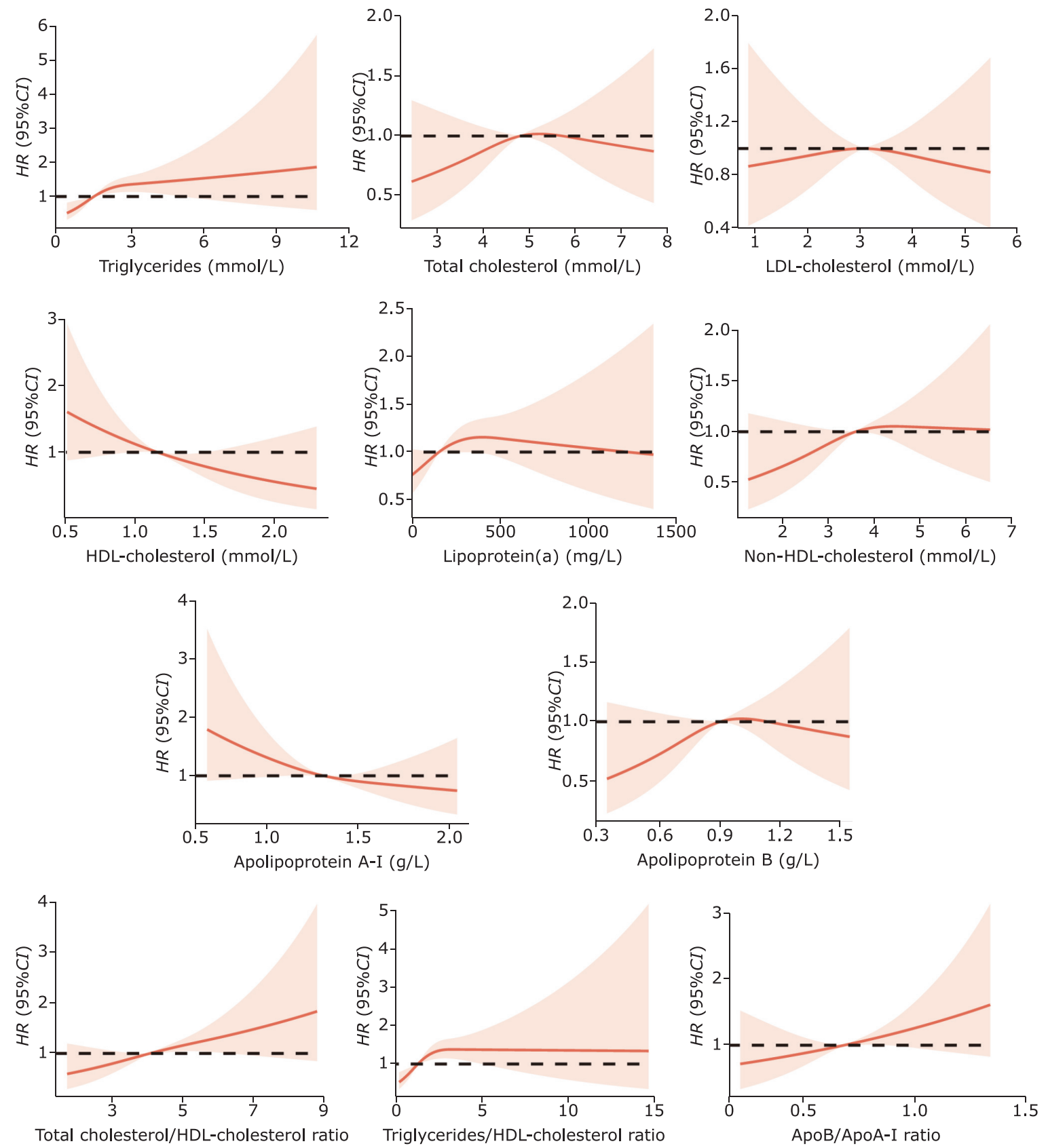

Figure 4. Risk of cardiac hypertrophy at different lipid concentrations.

The adjustment factors include age, sex, BMI, systolic blood pressure, red blood cell count, alanine transaminase, blood urea nitrogen, In(HOMA-IR), and the interaction between $\ln ($ HOMA-IR) and lipids.

the duration of follow-up, and excluding individuals who had hypertension at baseline. The results are shown in Supplementary Table S4. ApoB levels per SD increase did not result in a meaningful risk change in the sensitivity analysis, but this result was reasonable since the $A p o B$ increase per tertile was greater than the per SD. Other results observed in the sensitivity analyses were similar to the longitudinal cohort.

\section{DISCUSSION}

Based on a large Chinese population of overall 309,400 participants, we used the most comprehensive lipid profiles to date to explore the relationship between lipid markers and LVH in this study. We found that elevations in TG, lipoprotein(a), TC/HDL-C, and TG/HDL-c were associated with an increased risk of presenting LVH. In the longitudinal cohort, TG, non- 
Table 2. The final model determined by the forward stepwise selection (likelihood ratio) when different lipids were put into the Cox model together

\begin{tabular}{llc}
\hline \multicolumn{1}{c}{ Variables } & HR $(95 \% C I)^{\mathrm{a}}$ & $P$ value \\
\hline Age $(\mathrm{yrs})$ & $1.032(1.017,1.046)$ & $<0.001$ \\
Sex $(\mathrm{female} / \mathrm{male})$ & $0.352(0.239,0.519)$ & $<0.001$ \\
BMI $\left(\mathrm{kg} / \mathrm{m}^{2}\right)$ & $1.124(1.086,1.164)$ & $<0.001$ \\
Systolic blood pressure $(\mathrm{mm} \mathrm{Hg})$ & $1.026(1.020,1.033)$ & 0.001 \\
\hline Blood urea nitrogen $(\mathrm{mmol} / \mathrm{L})$ & $1.099(1.007,1.200)$ & 0.034 \\
Triglycerides & & 0.001 \\
\hline First tertile $(\leqslant 1.18 \mathrm{mmol} / \mathrm{L})$ & Reference & $<0.001$ \\
\hline Second tertile $(1.18-1.92 \mathrm{mmol} / \mathrm{L})$ & $1.827(1.277,2.614)$ & $1.904(1.336,2.714)$ \\
\hline Third tertile $(\geqslant 1.92 \mathrm{mmol} / \mathrm{L})$ & & \\
\hline
\end{tabular}

${ }^{a}$ In this Cox model, age, sex, BMI, systolic blood pressure, blood urea nitrogen, alanine transaminase, In(HOMA-IR), red blood cell count, triglycerides, HDL-cholesterol, non-HDL-cholesterol, apolipoprotein B, total cholesterol/HDL-cholesterol, triglycerides/HDL-cholesterol, and apolipoprotein B/apolipoprotein A-I were put into the initial model, and the final Cox model was determined using forward stepwise regression.

HDL-c, ApoB, TC/HDL-c, TG/HDL-c, and ApoB/ApoA-I increased were correlated with a higher risk of new-onset LVH. Elevated HDL-c was associated with both lower prevalence and new-onset of LVH. Furthermore, our study suggested TG had the most relevant association with new-onset LVH. These associations were independent of not only traditional cardiovascular risk factors (e.g., BMI, blood pressure), but also the role of insulin resistance. Although this evidence is preliminary, it reveals potential possibilities in the prevention and treatment of LVH by controlling circulating lipid levels.

The role of metabolism-related risk factors such as high BMI, diabetes, and hypertension in the development of LVH has been widely demonstrated ${ }^{[2]}$. However, although dyslipidemia is strongly correlated with other metabolic risk factors ${ }^{[29]}$, it is unclear whether it has an impact on LVH. With the increasing disease burden associated with dyslipidemia in recent years, it is important to clarify whether there is a correlation between dyslipidemia and $\mathrm{LVH}^{[4]}$. In previous studies, a positive relationship between increased plasma TG concentrations and the left ventricular mass or LVH was demonstrated in the European population ${ }^{[6,10]}$, and we further confirmed this association in the Chinese population with significantly larger sample size. Our study took more approaches to ensure the robustness of the results. We excluded individuals with coronary artery disease, structural heart disease, and those who had undergone cardiac surgery to reduce the potential influences from other cardiac diseases. In addition, we conducted an independent sensitivity analysis in a population without hypertension. Moreover, insulin resistance interplays with lipids and has a pivotal effect on cardiomyocytes' energy metabolism ${ }^{[1,28]}$. Therefore, in this study, HOMA-IR was used to reflect the degree of insulin resistance, and interaction between insulin resistance and blood lipids was controlled in the model analyzing the association between lipid and LVH. Using a longitudinal cohort, we further showed that baseline TG level proceeds the new onset of LVH. The results consistently supported the independent association between TG levels and LVH. However, whether a causal effect exists in high TG levels and LVH remains to be explored in the prospective setting.

The mechanism by which TGs cause LVH is not clear, but their function may be able to shed some light. TGs are an important form of fatty acid storage in the body, in which more than $90 \%$ of fatty acids are stored ${ }^{[30,31]}$. Under physiological conditions, fatty acids are the main energy resource for cardiac myocytes $^{[32]}$. Cardiac myocytes can take up fatty acids bound to plasma albumin as well as fatty acids stored as TG in chylomicrons or lipoproteins, with the latter being the major pathway ${ }^{[33]}$. Therefore, when TG levels are elevated, the supply of fatty acids will increase and thus cause an imbalance in fatty acid uptake and oxidation in cardiac myocytes. Excess fatty acids will be converted to TG, phospholipids, and other forms and stored in the cardiomyocytes ${ }^{[34]}$. Imaging analysis of the myocardium had also shown that lipid droplets could be observed in the myocardium of people with metabolic abnormalities ${ }^{[33]}$. Animal studies have 
shown that myocardial lipid deposition is associated with ventricular hypertrophy and dysfunction ${ }^{[35]}$. Thus, the adiposity of cardiomyocytes promoted by elevated TG may be an important cause of structural changes in the heart. In addition, current studies suggest that oxidation efficiency for fatty acids decreases during myocardial hypertrophy. However, the decrease in oxidative efficiency does not affect the uptake of fatty acids $^{[1]}$. Therefore, this would further exacerbate the imbalance between intracellular fatty acid supply and consumption. Animal studies have found that intracellularly stored TG may not cause toxicity ${ }^{[36,37]}$. Other lipid components converted from excess fatty acids may play a greater role, such as ceramide and diacylglycerol. The toxicity of these lipids can lead to mitochondrial dysfunction and apoptosis and may contribute to the progression of heart failure ${ }^{[34]}$. Also, patients with congenital fatty acid oxidation disorders lead to excessive lipid accumulation in cardiomyocytes, inducing a significantly increased incidence of sudden cardiac death in these patients ${ }^{[5]}$.

In recent years, the prevalence of dyslipidemia in China has been increasing ${ }^{[14,15]}$. It is noteworthy that high TG and low HDL-c are the main types of dyslipidemia in the Chinese population, in contrast to the predominance of high TC and high LDL-C in the United States and Europe ${ }^{[38]}$. This feature suggests that management of TG in the Chinese population may yield greater benefit. The role of TG in the development of cardiovascular disease has received increasing attention in recent years, yet the protective effect of TG control has been controversial ${ }^{[39,40]}$. Previous studies showed that Omega-3 improves left ventricle function, reduces recurrent heart failure, and reduces adverse left ventricle remodeling in patients with hypertriglyceridemia ${ }^{[41-44]}$. Because Omega-3 improves cardiac function may via multiple mechanisms, it is difficult to determine whether this effect is achieved directly by affecting TG levels ${ }^{[45]}$. Fibrates, another type of drug lowering circulating TG levels, demonstrate effects in reducing myocardial hypertrophy in animal studies ${ }^{[46,47]}$. Whether fibrates treatment results in beneficial effects in LVH warrant to be further study.

Whether elevated HDL-c level is negatively associated with LVH has been inconsistent in previous studies $^{[7,8,10]}$. Our study supports the negative association between these two entities. However, apolipoprotein A-I is the scaffolding for lipidation of HDL-C and the change did not associate with the presence of
LVH in this study ${ }^{[48]}$. This finding may indicate that the effect of HDL-C is not due to the reverse cholesterol transport function, but rather that HDL-C is a reflective indicator of TG levels, a conclusion that could be supported by the strong negative correlation exhibited by HDL-C and TG in our study. Regarding cholesterol, the evidence between cholesterol and LVH is still inconsistent ${ }^{[7-10]}$. Our study showed that LDL-C was not associated with the prevalence and the new onset of LVH. We noted that in the study by Aung et al, LDL-C showed a negative correlation with left ventricle mass in the observational results, despite the Mendelian randomization results indicating that LDL-C was associated with increased left ventricle mass. ${ }^{[10]}$ Therefore, the role of cholesterol, especially LDL-C, in LVH needs to be further confirmed in future studies. Meantime, the conclusions on the effect of statins on LVH are inconsistent in animal studies ${ }^{[49,50]}$. The association between LVH and heart failure is well established ${ }^{[2,51]}$, the latest ECS guideline for the treatment of heart failure stated that statins are not recommended in patients with heart failure without atherosclerotic cardiovascular disease because there is no evidence to support benefit ${ }^{[52]}$.

Limitation Several limitations should be mentioned in this study. First, our retrospective cohorts had inherent limitations in analyzing the causal relationship between lipid levels and LVH. Second, the study populations were based on health examinations but were not based on random sampling, and study data may underrepresent the rural populations and lower-income individuals. Third, the original images were not able to be reviewed independently by investigators, thus, interobserver variations have led to an increase in the deviation in the diagnose of LVH. Fourth, the mean follow-up period was relatively short for discovering enough population developing LVH. Fifth, the mean age of participants was around 50 in this study, which indicated this conclusion may be interpreted with caution when it extrapolates to a broader population.

Conclusion With the most comprehensive lipid profiles, our study provides new evidence for the view that an association exists between lipids and structural changes in the heart. In this study, TG and HDL-C demonstrated strong and stable associations with LVH. Therefore, the role played by lipids, especially TG, in adverse cardiac remodeling should be considered as well as further studied. 


\section{Supplementary material}

available at http://cmsj.cams.cn/EN/10.24920/004066.

Table S1. Correlation test (Spearman test) between lipid profiles.

Table S2. Characteristics of the national cross-sectional population.

Figure S1. Association of lipids and LVH in the national cross-sectional population.

Table S3. Baseline characteristics of the cohort.

Table S4. Results of sensitivity analysis for the longitudinal cohort.

\section{Conflict of interest}

The authors declare that the research was conducted in the absence of any commercial or financial relationships that could be construed as a potential conflict of interest.

\section{Author contributions}

All authors had full access to all of the data in the study. Huang XW and Deng KQ designed the study, collected, and analyzed data, and wrote the manuscript. Zhang $X Y$, Wang WX, Lin $L$, Zheng YM, Yao YG, Lu HM, Liu F, Chen $L D$, Zhang GL, Liu YP, and Yang $Q Y$ collected and reviewed the data. Qin JJ, Lei F, and Cai JJ provided valuable suggestions for study design and data analysis. Lu HM and She ZG contributed equally, designed the project, edited the manuscript, and supervised the study. All authors have approved the final version of this paper.

\section{Funding}

This work was supported by grants from the National Nature Science Foundation of China (82000386, 81970364, 82000299, 81870171, 82170436).

\section{REFERENCES}

1. Nakamura M, Sadoshima J. Mechanisms of physiological and pathological cardiac hypertrophy. Nat Rev Cardiol 2018; 15(7):387-407. doi: 10.1038/s41569-018-0007-y.

2. Gjesdal O, Bluemke DA, Lima JA. Cardiac remodeling at the population level-risk factors, screening, and outcomes. Nat Rev Cardiol 2011; 8(12):673-85. doi: 10.1038/nrcardio. 2011.154

3. Bluemke DA, Kronmal RA, Lima JAC, et al. The Relationship of Left Ventricular Mass and Geometry to Incident Cardiovascular Events. The MESA (Multi-Ethnic Study of Atherosclerosis) Study. J Am Coll Cardiol 2008; 52(25):2148-55. doi: 10.1016/j.jacc.2008.09.014.

4. Wang W, Hu M, Liu H, et al. Global Burden of Disease Study 2019 suggests that metabolic risk factors are the leading drivers of the burden of ischemic heart disease. Cell Metab
2021; 33(10):1943-56.e2. doi: 10.1016/j.cmet.2021.08.005.

5. Schulze PC, Drosatos K, Goldberg IJ. Lipid use and misuse by the heart. Circ Res 2016; 118(11):1736-51. doi: 10.1161/ CIRCRESAHA.116.306842.

6. Pietri $P$, Georgiopoulos $G$, Tsiachris $D$, et al. Triglycerides are related to left ventricular mass in hypertensive patients independently of other cardiometabolic risk factors: the effect of gender. Sci Rep 2020; 10(1):1-7. doi: 10.1038/s41598-02070237-1.

7. Horio T, Miyazato J, Kamide K, et al. Influence of low high-density lipoprotein cholesterol on left ventricular hypertrophy and diastolic function in essential hypertension. Am J Hypertens 2003; 16(11 I):938-44. doi: 10.1016/S08957061(03)01015-X.

8. Schillaci G, Vaudo G, Reboldi G, et al. Influence of low high-density lipoprotein cholesterol on left ventricular hypertrophy and diastolic function in essential hypertension. J Hypertens 2001; 19(12):2265-70. doi: 10.1097/00004872200112000-00021.

9. Jullien V, Gosse P, Ansoborlo P, et al. Relationship between left ventricular mass and serum cholesterol level in the untreated hypertensive. J Hypertens 1998; 16(7):1043-7. doi: 10.1097/00004872-199816070-00019.

10. Aung N, Sanghvi MM, Piechnik SK, et al. The effect of blood lipids on the left ventricle: a mendelian randomization study. J Am Coll Cardiol 2020; 76(21):2477-88. doi: 10.1016/j. jacc.2020.09.583.

11. Mehta A, Shapiro MD. Apolipoproteins in vascular biology and atherosclerotic disease. Nat Rev Cardiol 2021; 19(3):168-79. doi: 10.1038/s41569-021-00613-5.

12. Wang $H$, Li Z, Guo $X$, et al. The impact of nontraditional lipid profiles on left ventricular geometric abnormalities in general Chinese population. BMC Cardiovasc Disord 2018; 18(1):111. doi: $10.1186 / \mathrm{s} 12872-018-0829-x$.

13. Di Bonito $P$, Moio $N$, Scilla $C$, et al. Usefulness of the high triglyceride-to-HDL cholesterol ratio to identify cardiometabolic risk factors and preclinical signs of organ damage in outpatient children. Diabetes Care 2012; 35(1):158-62. doi: 10.2337/dc11-1456.

14. Taddei $\mathrm{C}$, Zhou B, Bixby $\mathrm{H}$, et al. Repositioning of the global epicentre of non-optimal cholesterol. Nature 2020; 582(7810):73-7. doi: 10.1038/s41586-020-2338-1.

15. Zhao D, Liu J, Wang $M$, et al. Epidemiology of cardiovascular disease in China: current features and implications. Nat Rev Cardiol 2019; 16(4):203-12. doi: 10.1038/s41569-0180119-4.

16. Zhao D. Epidemiological features of cardiovascular disease in Asia. JACC Asia 2021; 1(1):1-13. doi: 10.1016/j.jacasi. 2021.04.007.

17. Kario K, Chia Y, Sukonthasarn A, et al. Diversity of and initiatives for hypertension management in Asia-Why we need the HOPE Asia Network. J Clin Hypertens 2020; 22(3):331-43. doi: $10.1111 /$ jch.13733.

18. Kou S, Caballero L, Dulgheru R, et al. Echocardiographic reference ranges for normal cardiac chamber size: results 
from the NORRE study. Eur Hear J Cardiovasc Imaging 2014; 15(6):680-90. doi: 10.1093/ehjci/jet284.

19. Sun NL, Jaw-Wen C, Wang JG, et al. Expert consensus on diagnosis and treatment of hypertension complicated with left ventricular hypertrophy in Asian. Chin J Hypertens 2016; 24(7):619-27. doi: 10.16439/j.cnki.1673-7245.2016.07.008.

20. Joint Committee Issued Chinese Guideline for the Management of Dyslipidemia in Adults. 2016 Chinese Guideline for the Management of Dyslipidemia in Adults. Zhonghua Xin Xue Guan Bing Za Zhi 2016; 44(10):833-53. doi: 10.3760/cma. j.issn.0253-3758.2016.10.005.

21. Wu Y. Overweight and obesity in China. BMJ 2006; 333(7564):362-3. doi: 10.1136/bmj.333.7564.362.

22. Joint Committee for Guideline Revision. 2018 Chinese Guidelines for Prevention and Treatment of Hypertension-A report of the Revision Committee of Chinese Guidelines for Prevention and Treatment of Hypertension. J Geriatr Cardiol 2019; 16(3):182-241. doi: 10.11909/ j.issn.1671-5411.2019.03.014.

23. Chinese Elderly Type 2 Diabetes Prevention and Treatment of Clinical Guidelines Writing Group, Geriatric Endocrinology and Metabolism Branch of Chinese Geriatric Society, Geriatric Endocrinology and Metabolism Branch of Chinese Geriatric Health Care Society, Geriatric Professional Committee of Beijing Medical Award Foundation, National Clinical Medical Research Center for Geriatric Diseases (PLA General Hospital). Clinical guidelines for prevention and treatment of type 2 diabetes mellitus in the elderly in China (2022 edition). Zhonghua Nei Ke Za Zhi 2022; 61(1):12-50. Chinese. doi: 10.3760/cma.j.cn112138-20211027-00751.

24. Matthews DR, Hosker JP, Rudenski AS, et al. Homeostasis model assessment: insulin resistance and $\beta$-cell function from fasting plasma glucose and insulin concentrations in man. $\mathrm{Di}$ abetologia 1985; 28(7):412-9. doi: 10.1007/BF00280883.

25. Zhu L, She ZG, Cheng $X$, et al. Association of blood glucose control and outcomes in patients with COVID-19 and pre-existing type 2 diabetes. Cell Metab 2020; 31(6):1068-77.e3. doi: 10.1016/j.cmet.2020.04.021.

26. Waljee AK, Mukherjee A, Singal AG, et al. Comparison of imputation methods for missing laboratory data in medicine. BMJ Open 2013; 3(8):e002847. doi: 10.1136/bmjopen2013-002847.

27. Savage DB, Petersen KF, Shulman GI. Disordered lipid metabolism and the pathogenesis of insulin resistance. Physiol Rev 2007; 87(2):507-20. doi: 10.1152/physrev.00024.2006.

28. Ng ACT, Delgado V, Borlaug BA, et al. Diabesity: the combined burden of obesity and diabetes on heart disease and the role of imaging. Nat Rev Cardiol 2021; 18(4):291-304. doi: 10.1038/s41569-020-00465-5.

29. Pirillo A, Casula M, Olmastroni E, et al. Global epidemiology of dyslipidaemias. Nat Rev Cardiol 2021; 18(10):689-700. doi: 10.1038/s41569-021-00541-4.

30. Shang R, Rodrigues $B$. Lipoprotein lipase and its delivery of fatty acids to the heart. Biomolecules 2021; 11(7):1-11. doi: 10.3390/biom 11071016
31. Zechner R, Madeo F, Kratky D. Cytosolic lipolysis and lipophagy: two sides of the same coin. Nat Rev Mol Cell Biol 2017; 18(11):671-84. doi: 10.1038/nrm.2017.76

32. Stanley WC, Recchia FA, Lopaschuk GD. Myocardial substrate metabolism in the normal and failing heart. Physiol Rev 2005; 85(3):1093-129. doi: 10.1152/physrev.00006.2004.

33. Goldberg IJ, Trent CM, Schulze PC. Lipid metabolism and toxicity in the heart. Cell Metab 2012; 15(6):805-12. doi: 10.1016/j.cmet.2012.04.006.

34. Bertero $E$, Maack C. Metabolic remodelling in heart failure. Nat Rev Cardiol 2018; 15(8):457-70. doi: 10.1038/s41569018-0044-6.

35. Szczepaniak LS, Victor RG, Orci L, et al. Forgotten but not gone: the rediscovery of fatty heart, the most common unrecognized disease in America. Circ Res 2007; 101(8):759-67. doi: 10.1161/CIRCRESAHA.107.160457.

36. Liu L, Shi X, Bharadwaj KG, et al. DGAT1 expression increases heart triglyceride content but ameliorates lipotoxicity. J Biol Chem 2009; 284(52):36312-23. doi: 10.1074/jbc. M109.049817.

37. Listenberger LL, Han X, Lewis SE, et al. Triglyceride accumulation protects against fatty acid-induced lipotoxicity. Proc Natl Acad Sci 2003; 100(6):3077-82. doi: 10.1073/ pnas. 0630588100 .

38. Lu Y, Zhang H, Lu J, et al. Prevalence of dyslipidemia and availability of lipid-lowering medications among primary health care settings in China. JAMA Netw Open 2021; 4(9):e2127573. doi: 10.1001/jamanetworkopen.2021.27573.

39. Libby $P$. The changing landscape of atherosclerosis. Nature 2021; 592(7855):524-33. doi: 10.1038/s41586-021-03392-8.

40. Raposeiras-Roubin S, Rosselló X, Oliva B, et al. Triglycerides and residual atherosclerotic risk. J Am Coll Cardiol 2021; 77(24):3031-41. doi: 10.1016/j.jacc.2021.04.059.

41. Bhatt DL, Steg PG, Miller M, et al. Cardiovascular risk reduction with icosapent ethyl for hypertriglyceridemia. N Engl J Med 2019; 380(1):11-22. doi: 10.1056/nejmoa1812792.

42. Heydari B, Abdullah S, Pottala JV, et al. Effect of omega-3 acid ethyl esters on left ventricular remodeling after acute myocardial infarction. Circulation 2016; 134(5):378-91. doi: 10.1161/CIRCULATIONAHA.115.019949.

43. Djoussé $L$, Cook NR, Kim E, et al. Supplementation with vitamin $\mathrm{D}$ and omega-3 fatty acids and incidence of heart failure hospitalization: vital-heart failure. Circulation 2020; 141(9): 784-6. doi: 10.1161/CIRCULATIONAHA.119.044645.

44. Campos-Staffico AM, Costa APR, Carvalho LSF, et al. Omega-3 intake is associated with attenuated inflammatory response and cardiac remodeling after myocardial infarction. Nutr J 2019; 18(1):1-8. doi: 10.1186/s12937-019-0455-1.

45. Block RC, Liu L, Herrington DM, et al. Predicting risk for incident heart failure with omega-3 fatty acids: from MESA. JACC Hear Fail 2019; 7(8):651-61. doi: 10.1016/ j.jchf.2019.03.008

46. Zou J, Le K, Xu S, et al. Fenofibrate ameliorates cardiac hypertrophy by activation of peroxisome proliferator-activated receptor-a partly via preventing p65-NFKB binding to NFATc4. 
Mol Cell Endocrinol 2013; 370(1-2):103-12. doi: 10.1016/ j.mce.2013.03.006.

47. Zhuang L, Mao Y, Liu Z, et al. FABP3 deficiency exacerbates metabolic derangement in cardiac hypertrophy and heart failure via PPARa pathway. Front Cardiovasc Med 2021; 8:722908. doi: 10.3389/fcvm.2021.722908.

48. Pownall HJ, Rosales C, Gillard BK, et al. High-density lipoproteins, reverse cholesterol transport and atherogenesis. Nat Rev Cardiol 2021; 18(10):712-23. doi: 10.1038/s41569-02100538-z.

49. Patel R, Nagueh SF, Tsybouleva N, et al. Simvastatin induces regression of cardiac hypertrophy and fibrosis and improves cardiac function in a transgenic rabbit model of human hypertrophic cardiomyopathy. Circulation 2001; 104(3):317-24. doi: $10.1161 /$ hc2801.094031.

50. Chang SA, Kim YJ, Lee HW, et al. Effect of rosuvastatin on cardiac remodeling, function, and progression to heart failure in hypertensive heart with established left ventricular hypertrophy. Hypertension 2009; 54(3):591-7. doi: 10.1161/ HYPERTENSIONAHA.109.131243.

51. Kawel-Boehm N, Kronmal R, Eng J, et al. Left ventricular mass at MRI and long-term risk of cardiovascular events: the multi-ethnic study of atherosclerosis (MESA). Radiology 2019; 293(1):107-14. doi: 10.1148/radiol.2019182871.

52. McDonagh TA, Metra M, Adamo M, et al. 2021 ESC Guidelines for the diagnosis and treatment of acute and chronic heart failure. Eur Heart J 2021; 42(36):3599-726. doi: 10.1093/ eurheartj/ehab368. 